Supporting information for:

\title{
Development of water solubility of 2-phenylsulfanylhydroquinone dimer dye
}

Akio Kamimura, ${ }^{*}, 1$ Haruka Umemoto, ${ }^{1}$ Takuji Kawamoto, ${ }^{1}$ and Takeshi Honda*,2

${ }^{1}$ Department of Applied Chemistry, Yamaguchi University, Ube 755-8611, Japan

${ }^{2}$ Department of Pharmacology, School of Medicine, Yamaguchi University, Ube 755-8505, Japan

\section{Contents}

${ }^{1} \mathrm{H}$ and ${ }^{13} \mathrm{C}$ NMR spectra for compounds $\quad$ S2

UV and PL spectra for compounds $\quad$ S12 

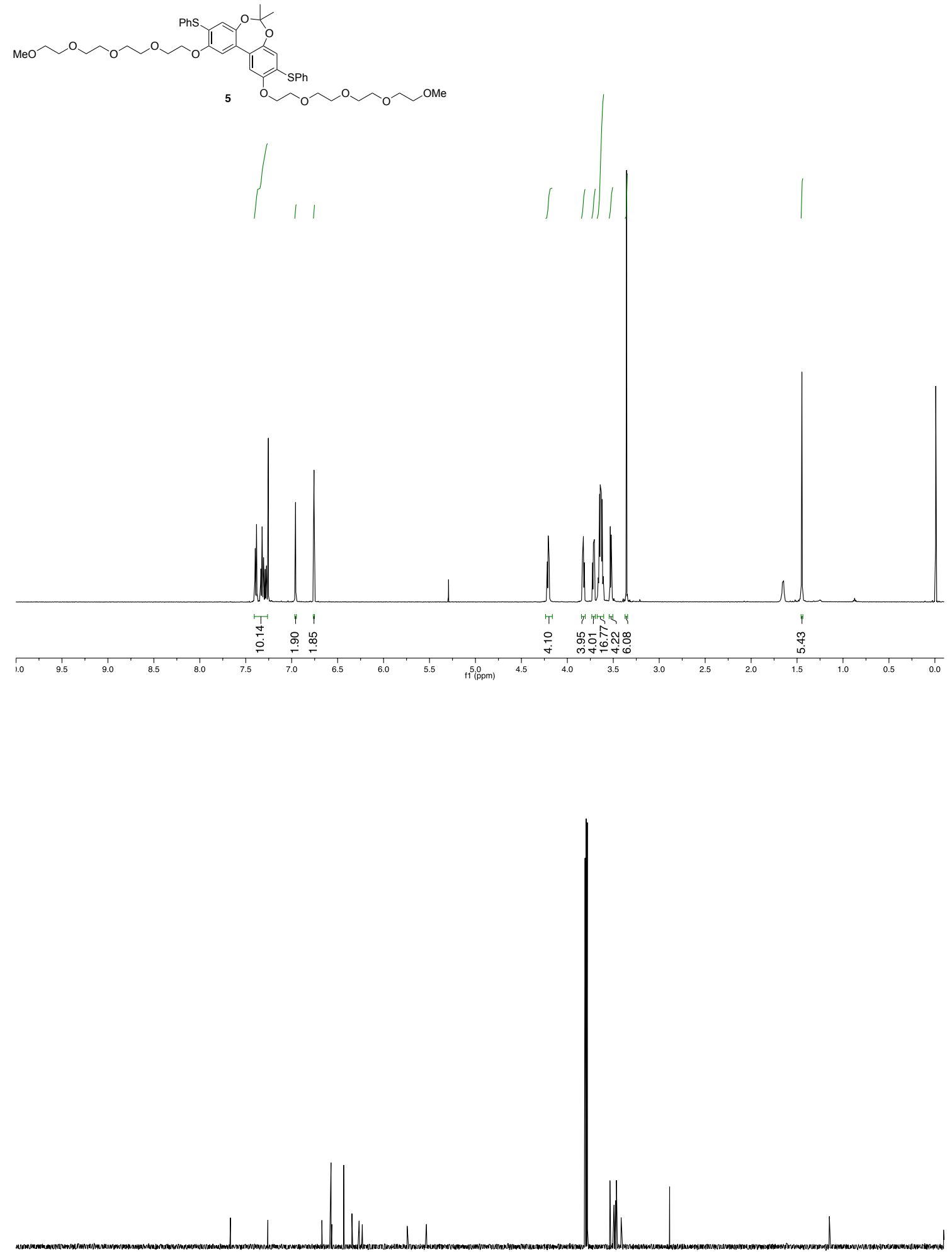

Figure S1. ${ }^{1} \mathrm{H}$ and ${ }^{13} \mathrm{C}$ NMR spectra for compound 5. 

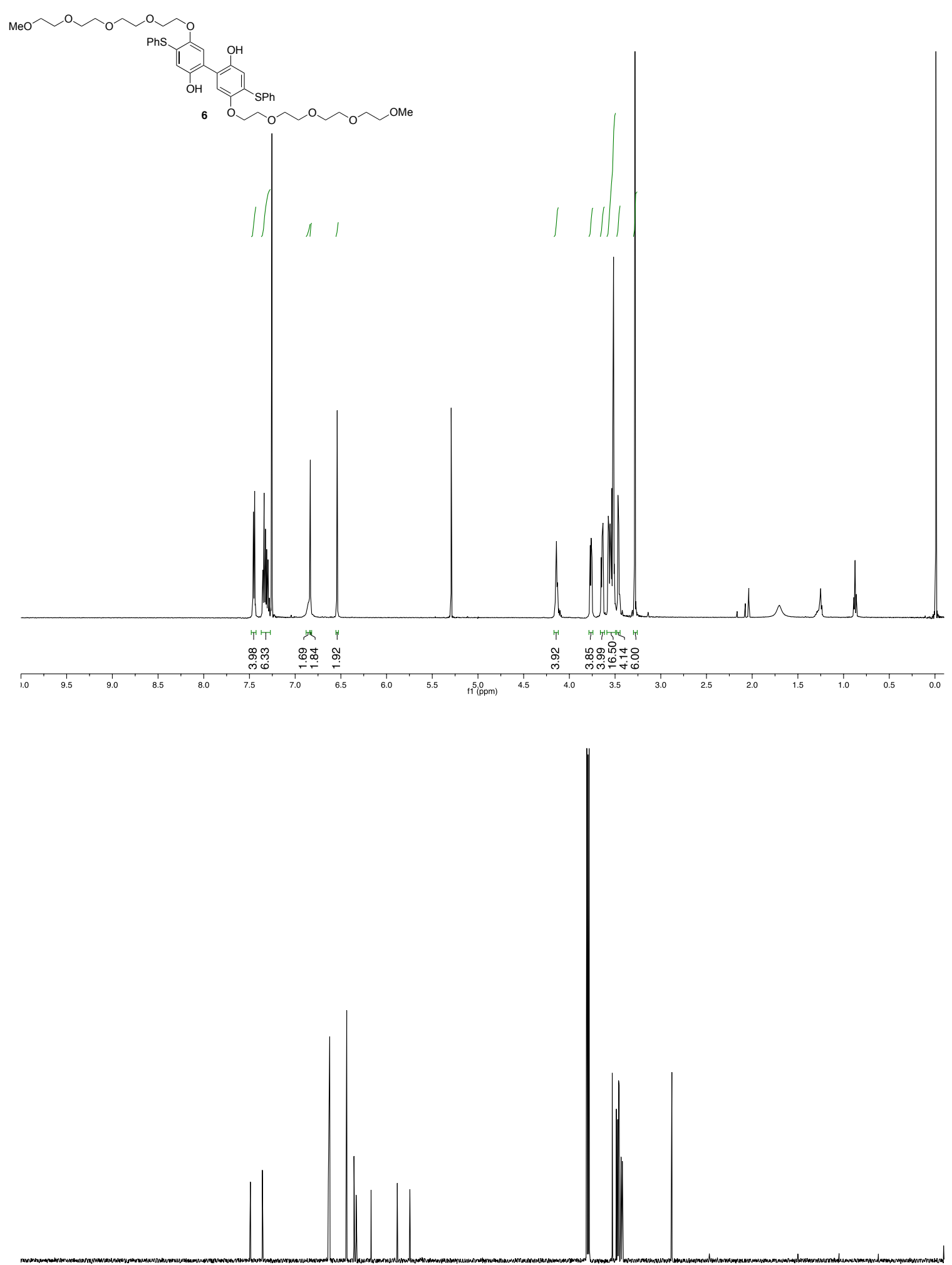

Figure S2. ${ }^{1} \mathrm{H}$ and ${ }^{13} \mathrm{C}$ NMR spectra for compound 6. 

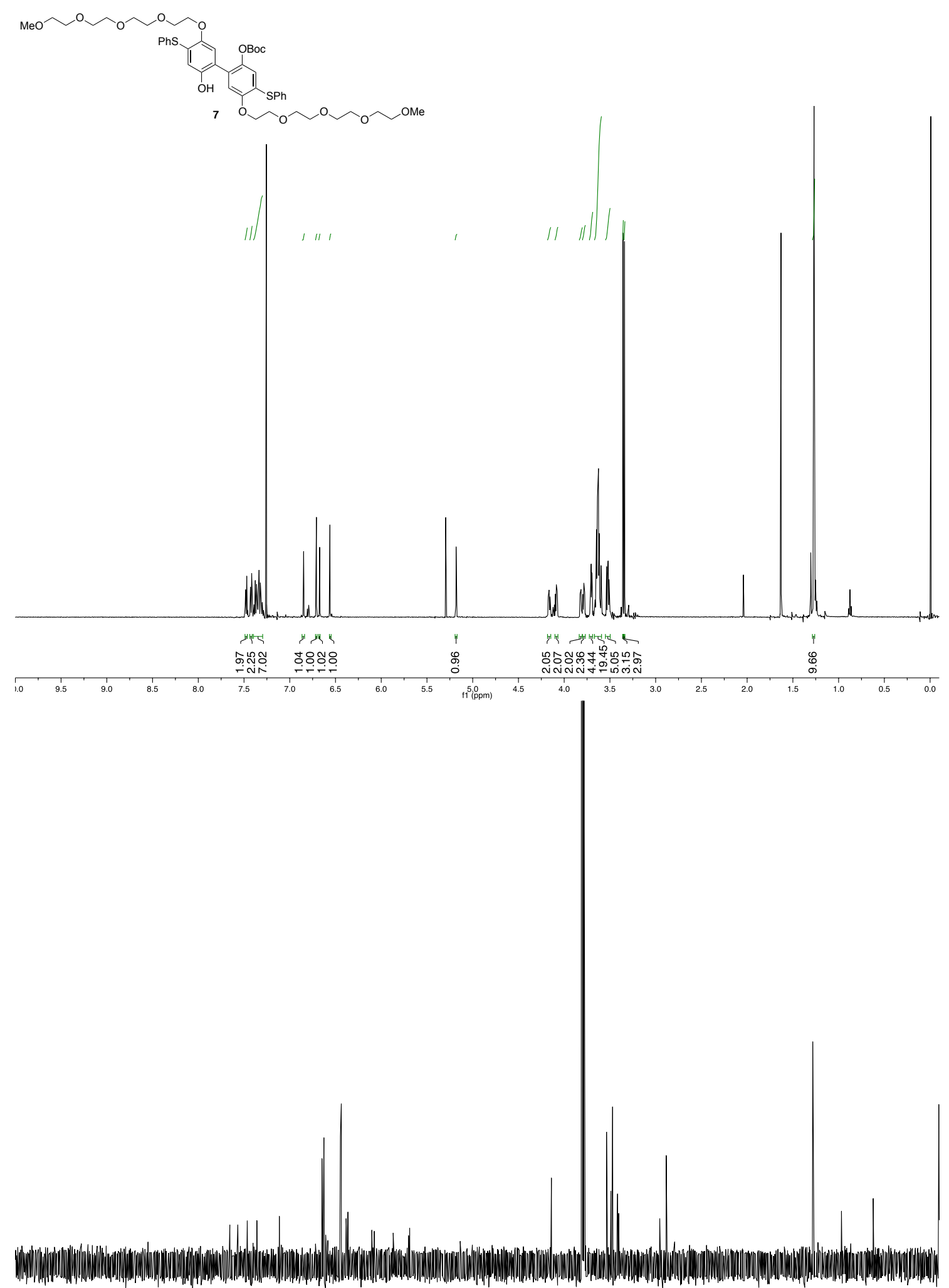

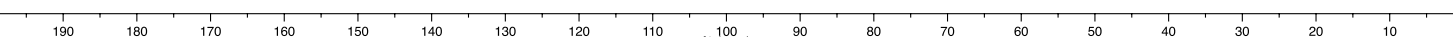

Figure S3. ${ }^{1} \mathrm{H}$ and ${ }^{13} \mathrm{C}$ NMR spectra for compound 7. 

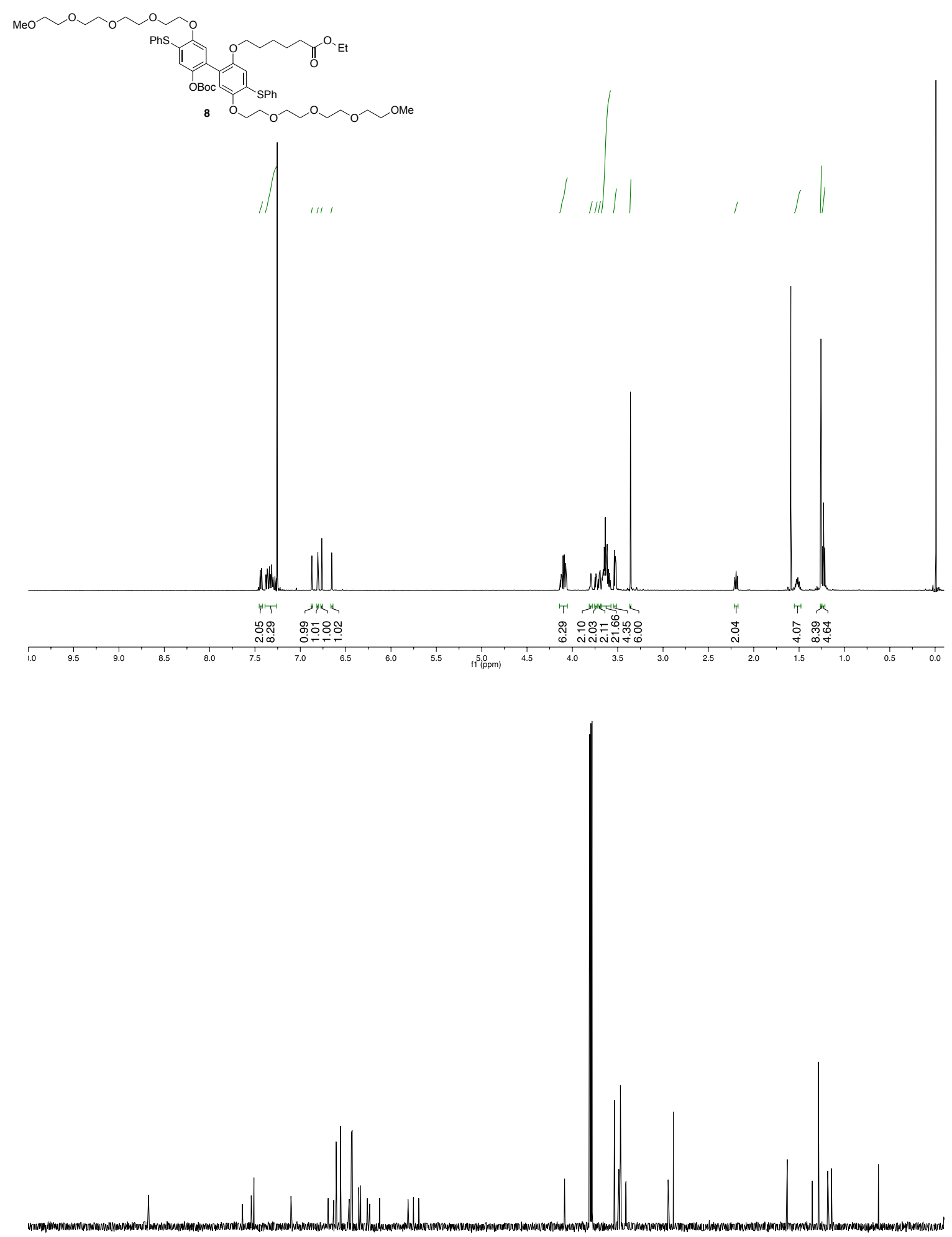

Figure S4. ${ }^{1} \mathrm{H}$ and ${ }^{13} \mathrm{C}$ NMR spectra for compound 8. 

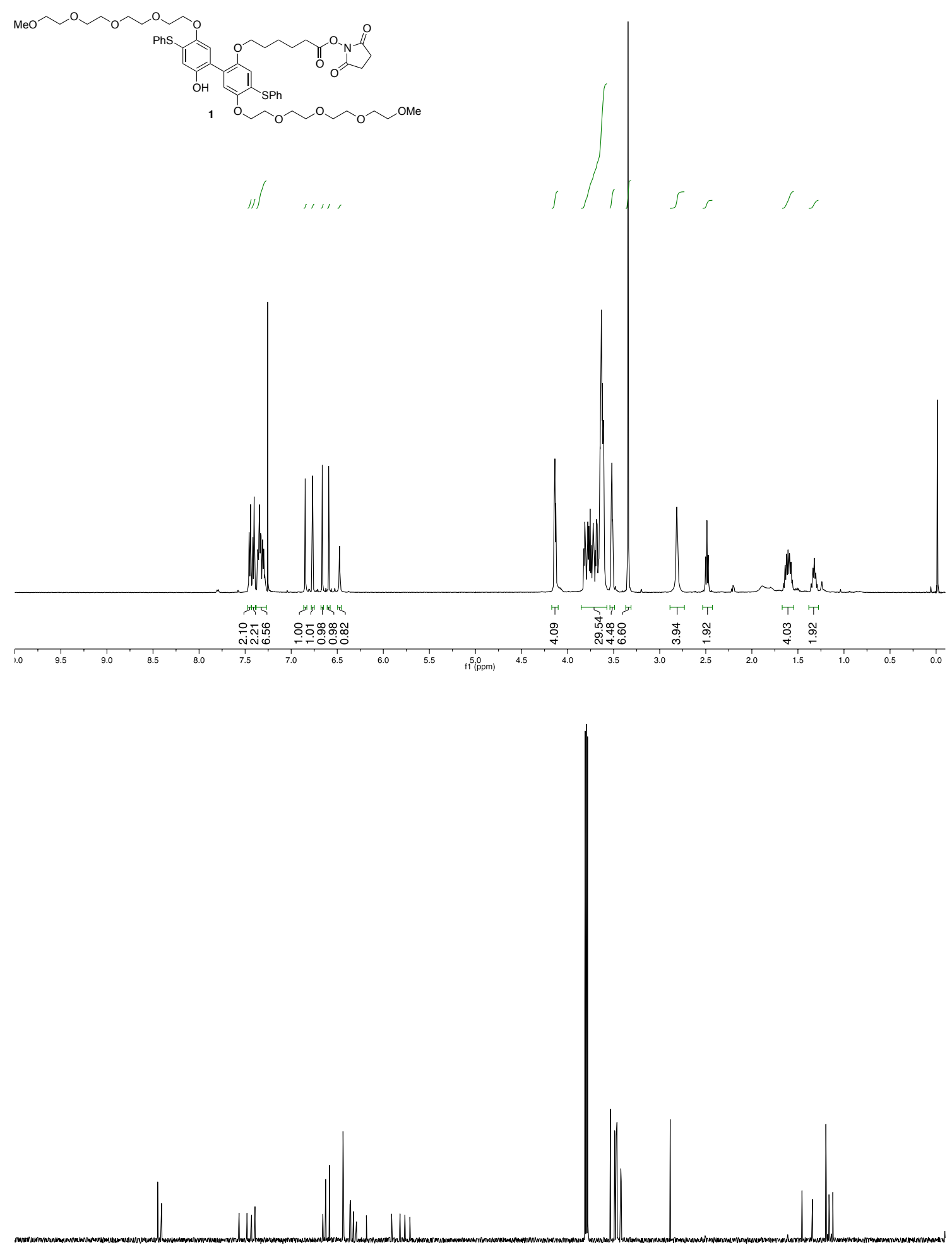

Figure S5. ${ }^{1} \mathrm{H}$ and ${ }^{13} \mathrm{C}$ NMR spectra for compound 1. 

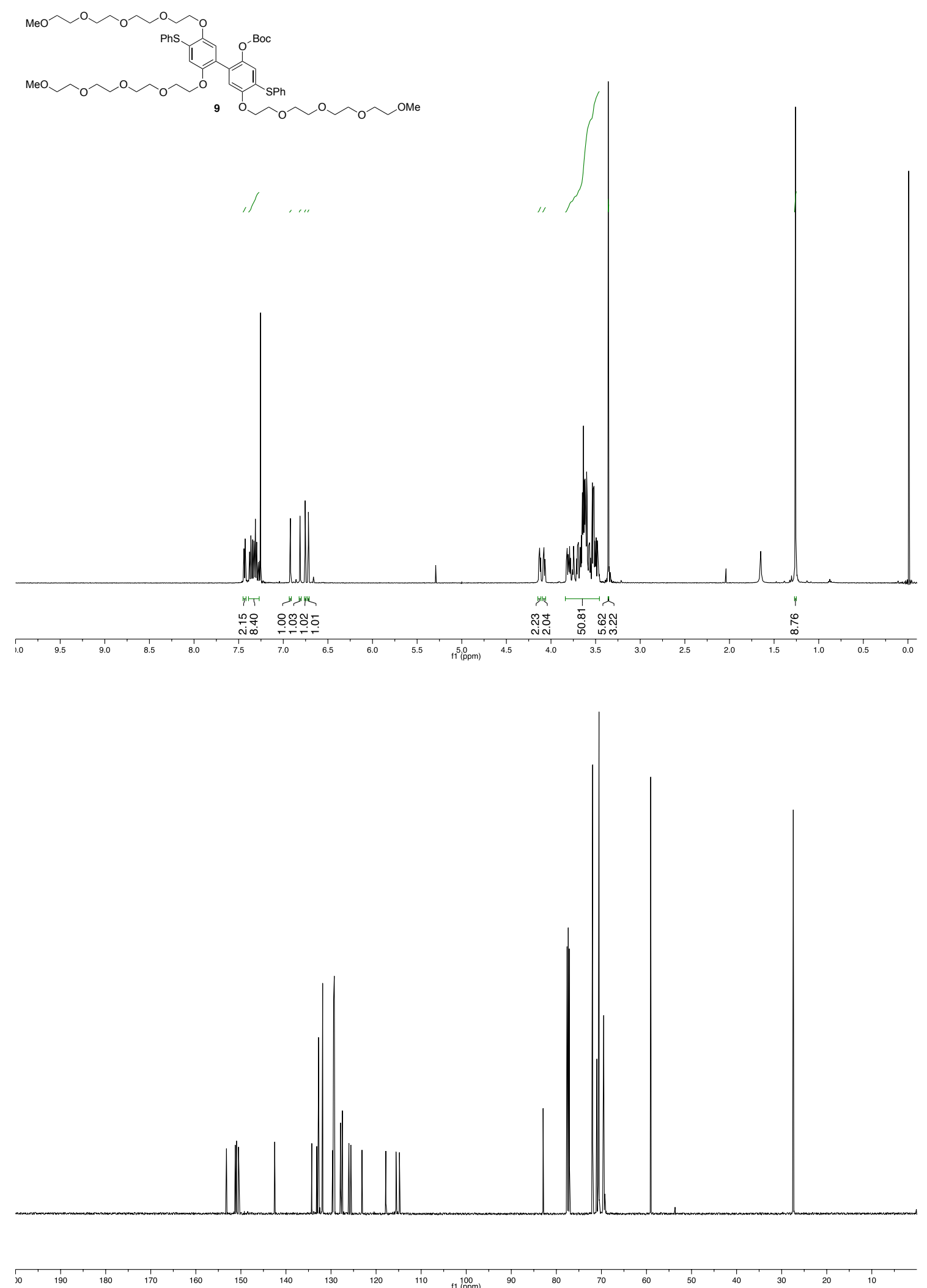

Figure S6. ${ }^{1} \mathrm{H}$ and ${ }^{13} \mathrm{C}$ NMR spectra for compound 9. 

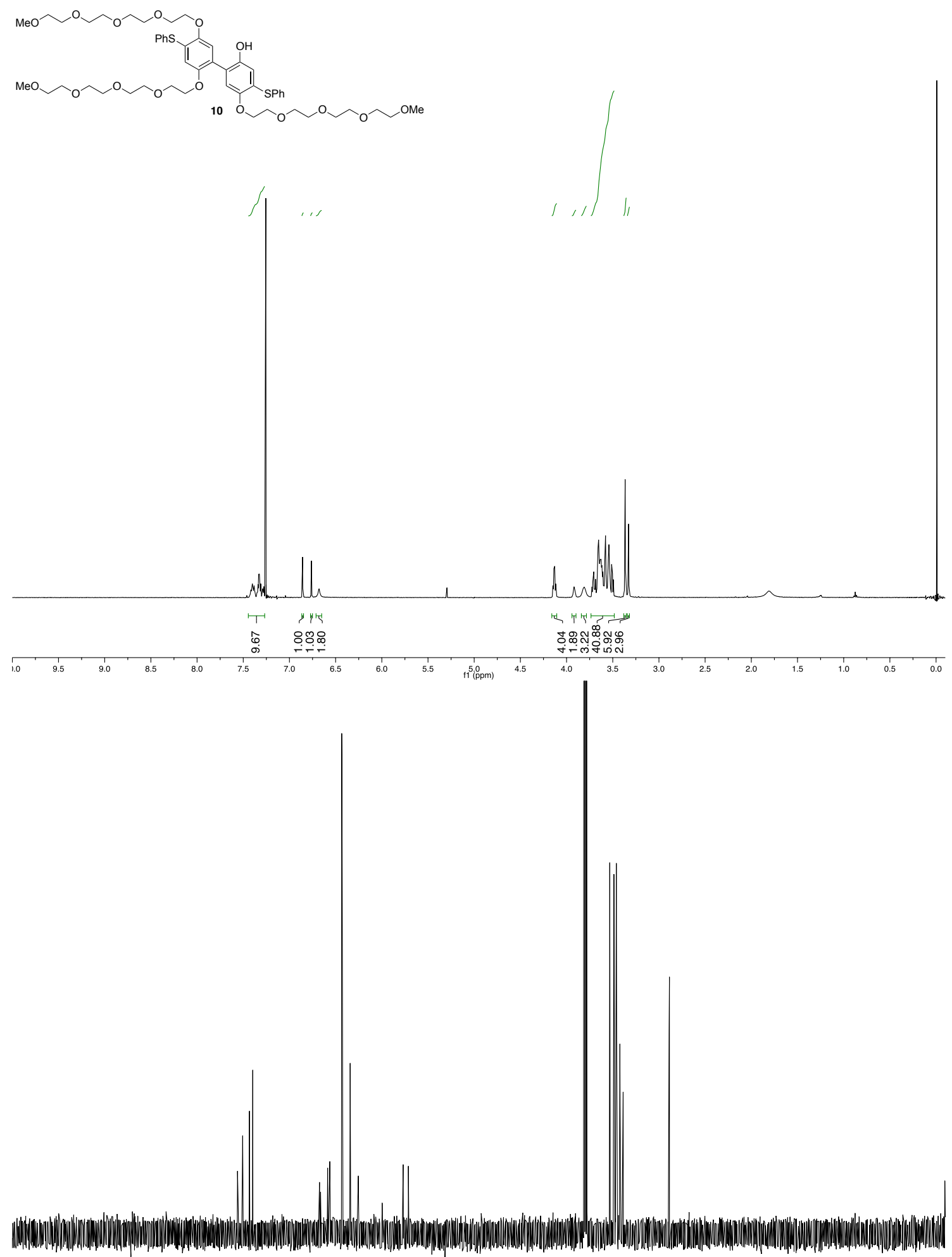

Figure S7. ${ }^{1} \mathrm{H}$ and ${ }^{13} \mathrm{C}$ NMR spectra for compound 10. 

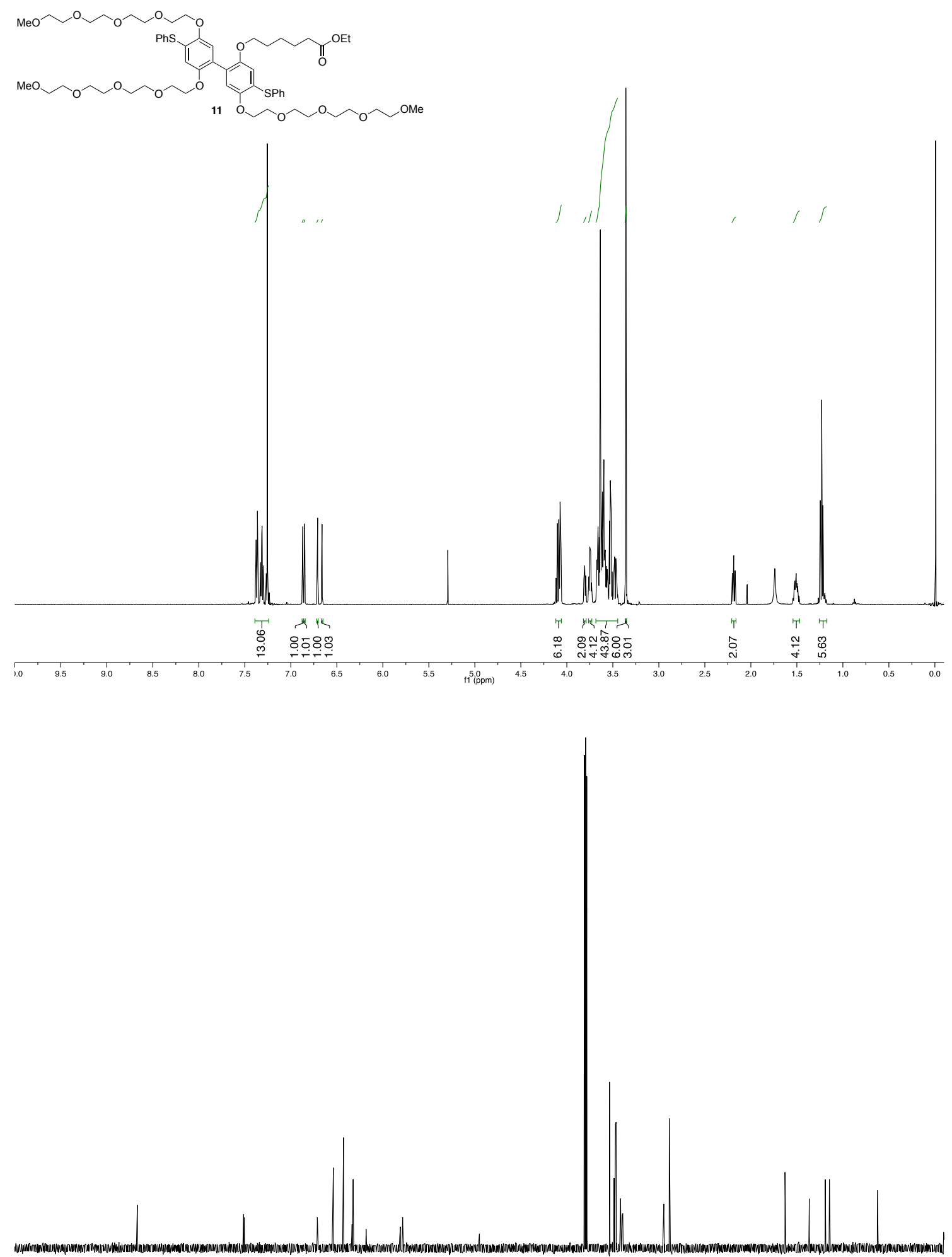

Figure S8. ${ }^{1} \mathrm{H}$ and ${ }^{13} \mathrm{C}$ NMR spectra for compound 11. 

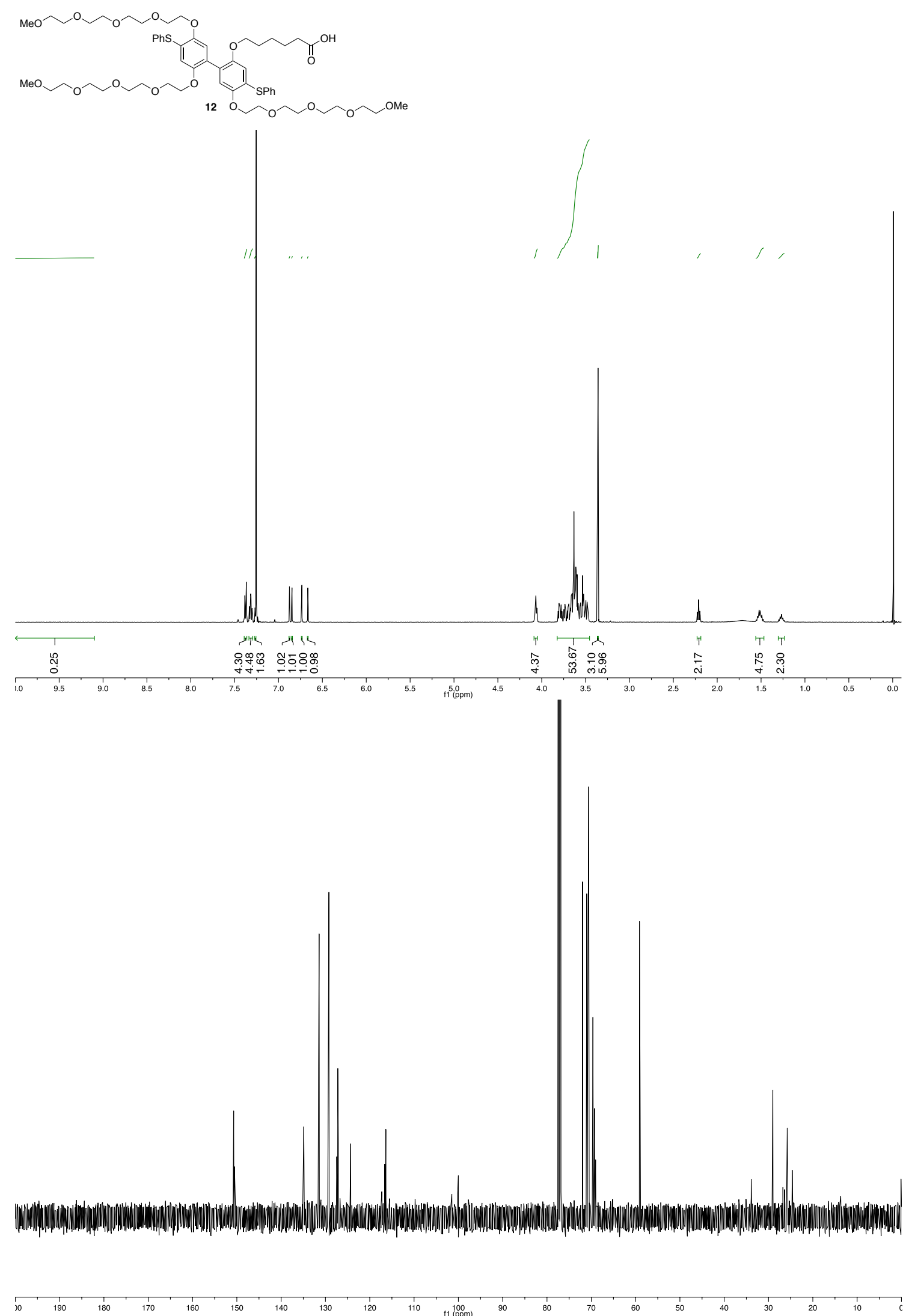

Figure S9. ${ }^{1} \mathrm{H}$ and ${ }^{13} \mathrm{C}$ NMR spectra for compound 12. 

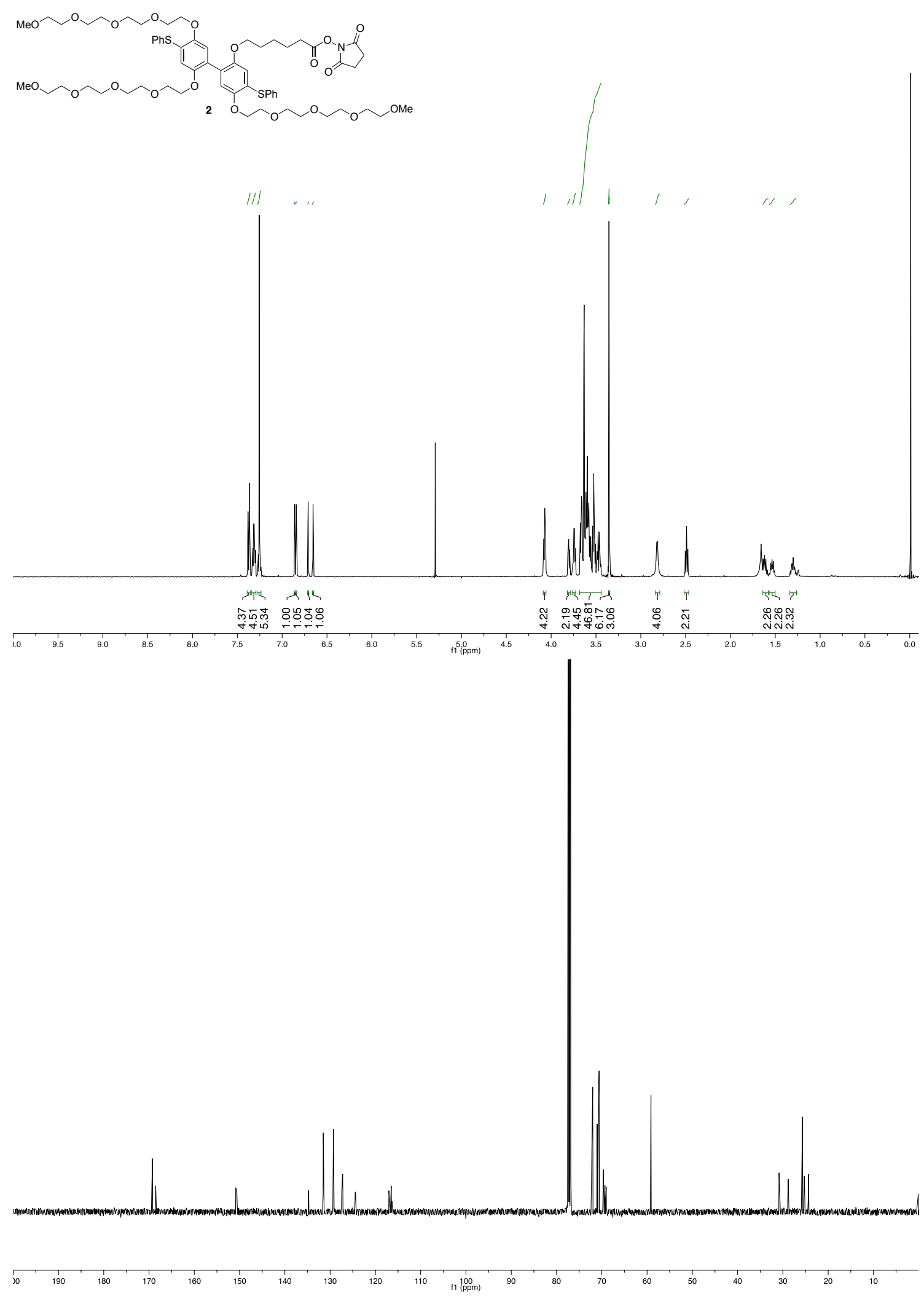

Figure S10. ${ }^{1} \mathrm{H}$ and ${ }^{13} \mathrm{C}$ NMR spectra for compound 2.

S 11 


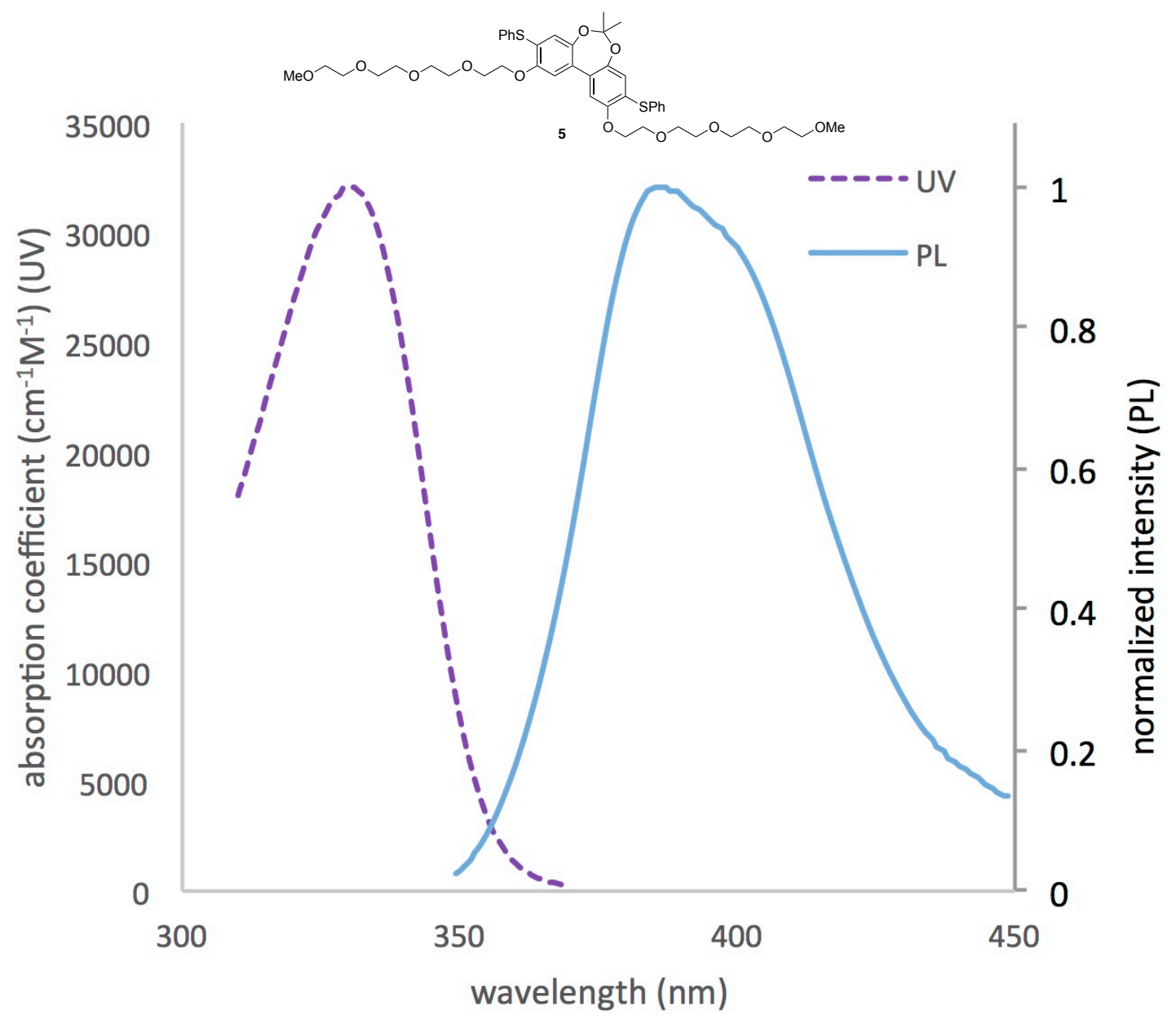

Figure S11. UV-PL spectra for compound 5 (in $\mathrm{MeOH}$ )

$\lambda_{\max }=330 \mathrm{~nm}, \varepsilon=3.2 \times 10^{4} \mathrm{M}^{-1} \mathrm{~cm}^{-1}, \lambda_{\mathrm{em}}=386 \mathrm{~nm}, \Phi=0.122$ 


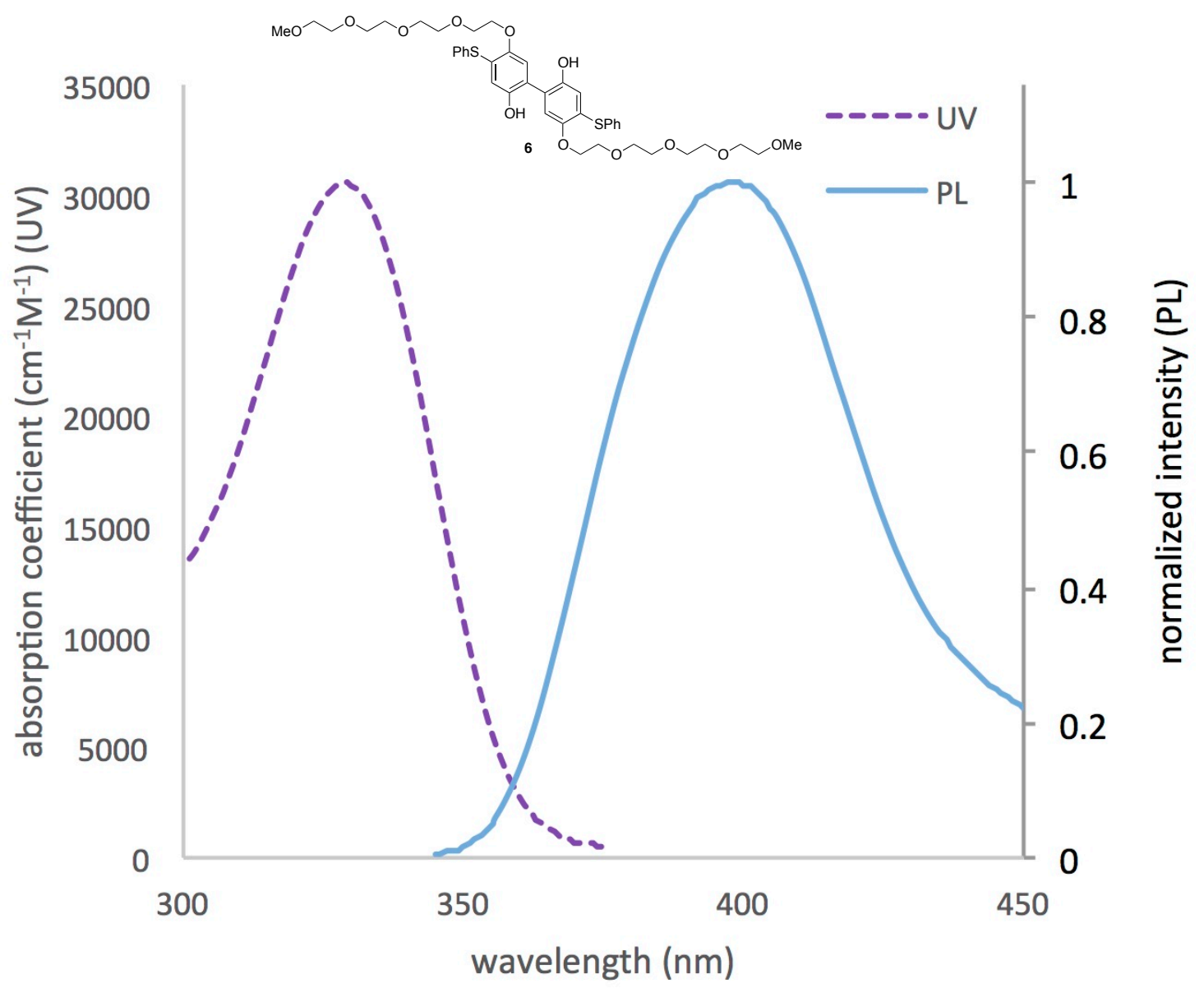

Figure S12. UV-PL spectra for compound 6 (in $\mathrm{MeOH}$ )

$\lambda_{\text {max }}=329 \mathrm{~nm}, \varepsilon=2.9 \times 10^{4} \mathrm{M}^{-1} \mathrm{~cm}^{-1}, \lambda_{\text {em }}=398 \mathrm{~nm}, \Phi=0.226$ 


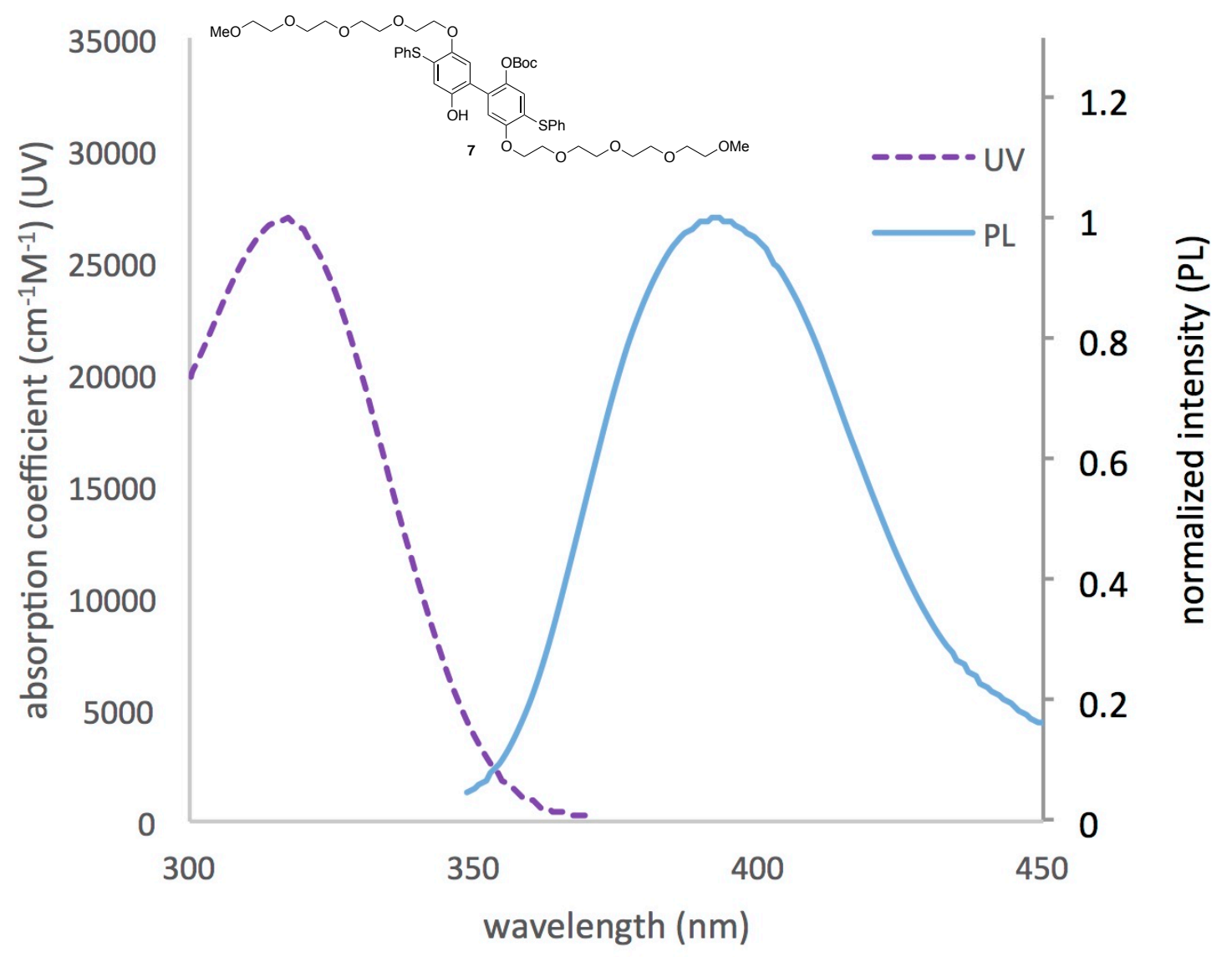

Figure S13. UV-PL spectra for compound 7 (in $\mathrm{MeOH}$ )

$\lambda_{\max }=317 \mathrm{~nm}, \varepsilon=2.7 \times 10^{4} \mathrm{M}^{-1} \mathrm{~cm}^{-1}, \lambda_{\mathrm{em}}=392 \mathrm{~nm}, \Phi=0.141$ 


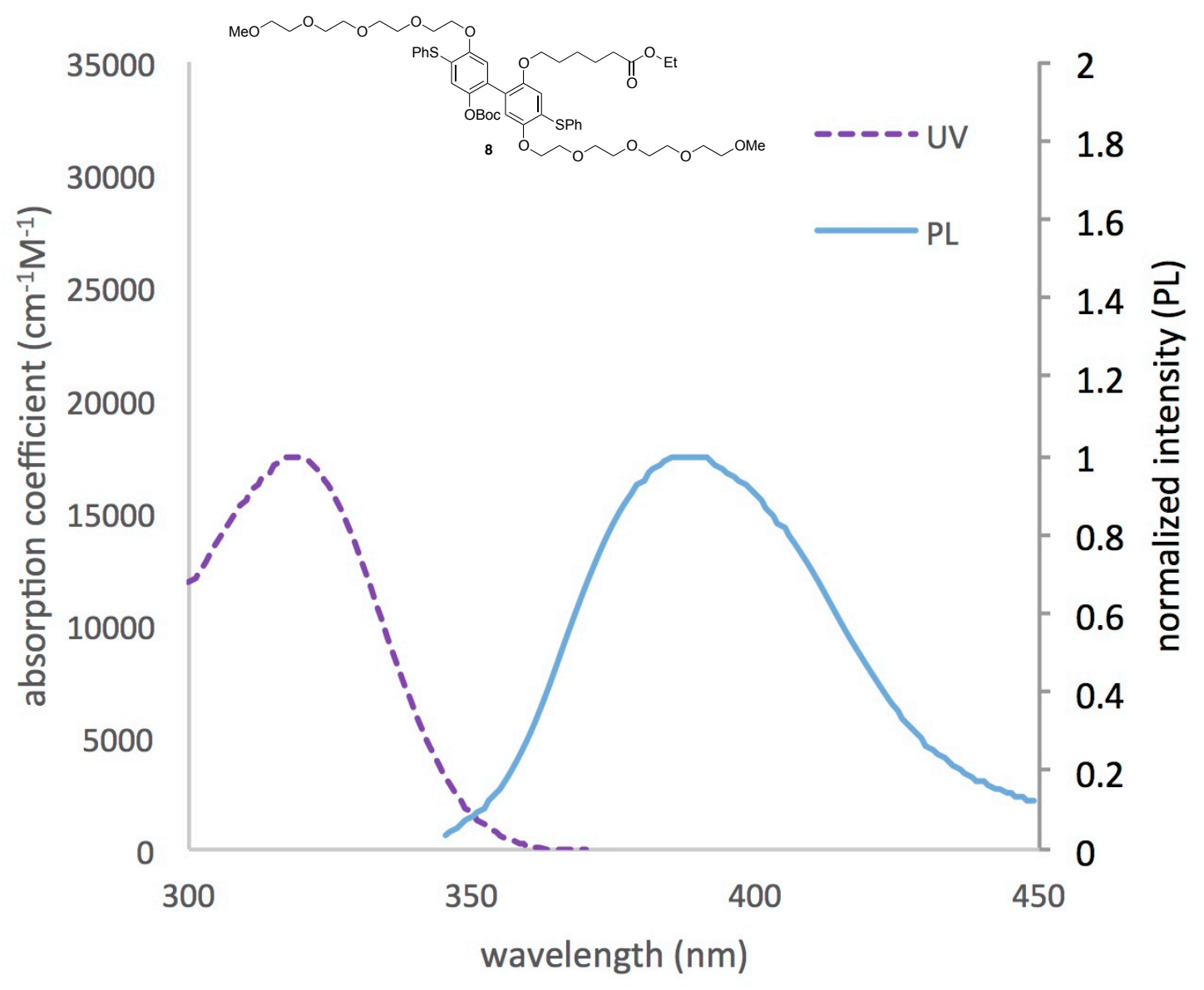

Figure S14. UV-PL spectra for compound 8 (in $\mathrm{MeOH}$ )

$\lambda_{\max }=318 \mathrm{~nm}, \varepsilon=1.8 \times 10^{4} \mathrm{M}^{-1} \mathrm{~cm}^{-1}, \lambda_{\mathrm{em}}=388 \mathrm{~nm}, \Phi=0.147$ 


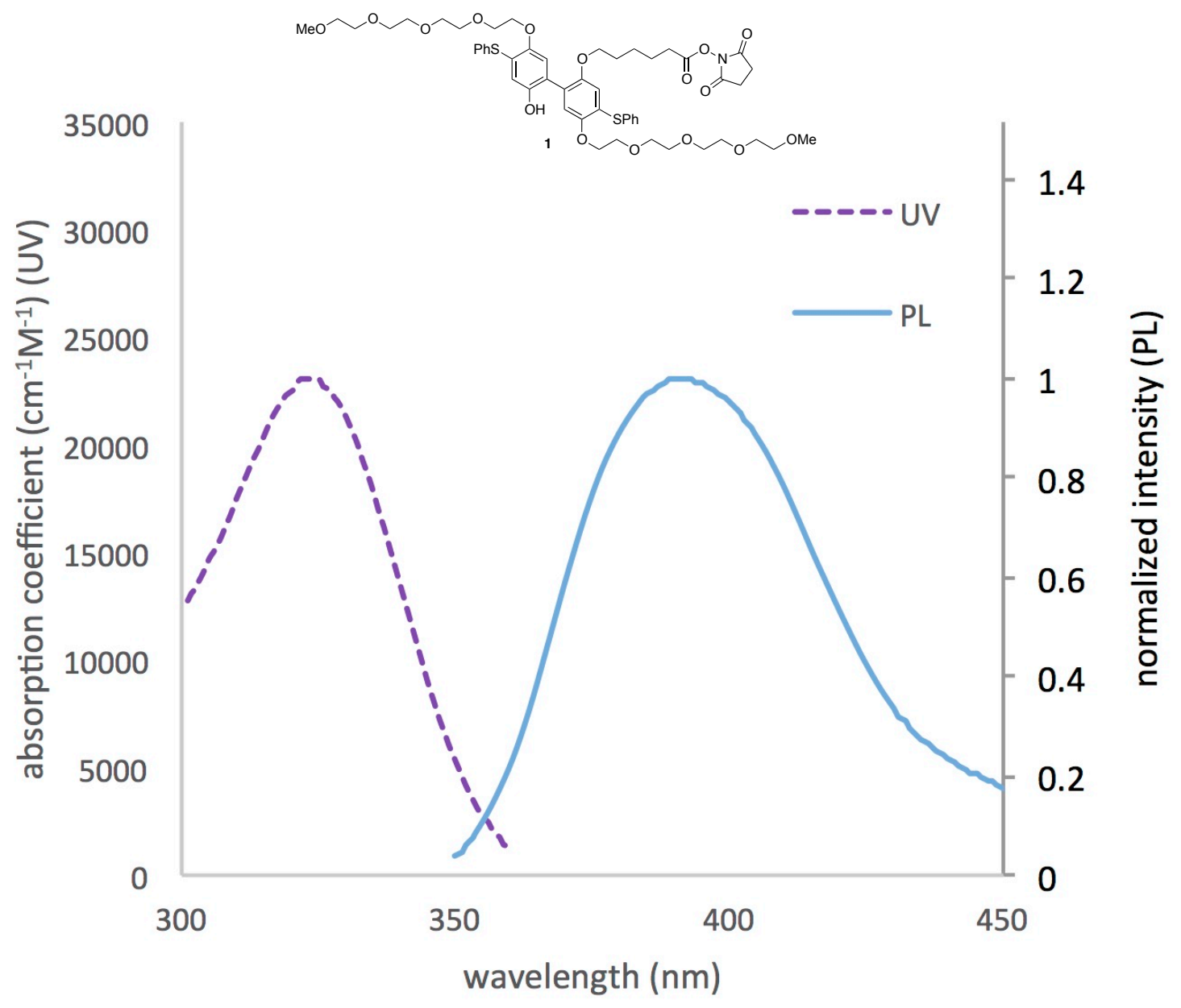

Figure S15. UV-PL spectra for compound 1 (in $\mathrm{MeOH}$ )

$\lambda_{\max }=324 \mathrm{~nm}, \varepsilon=2.4 \times 10^{4} \mathrm{M}^{-1} \mathrm{~cm}^{-1}, \lambda_{\mathrm{em}}=391 \mathrm{~nm}, \Phi=0.158$ 


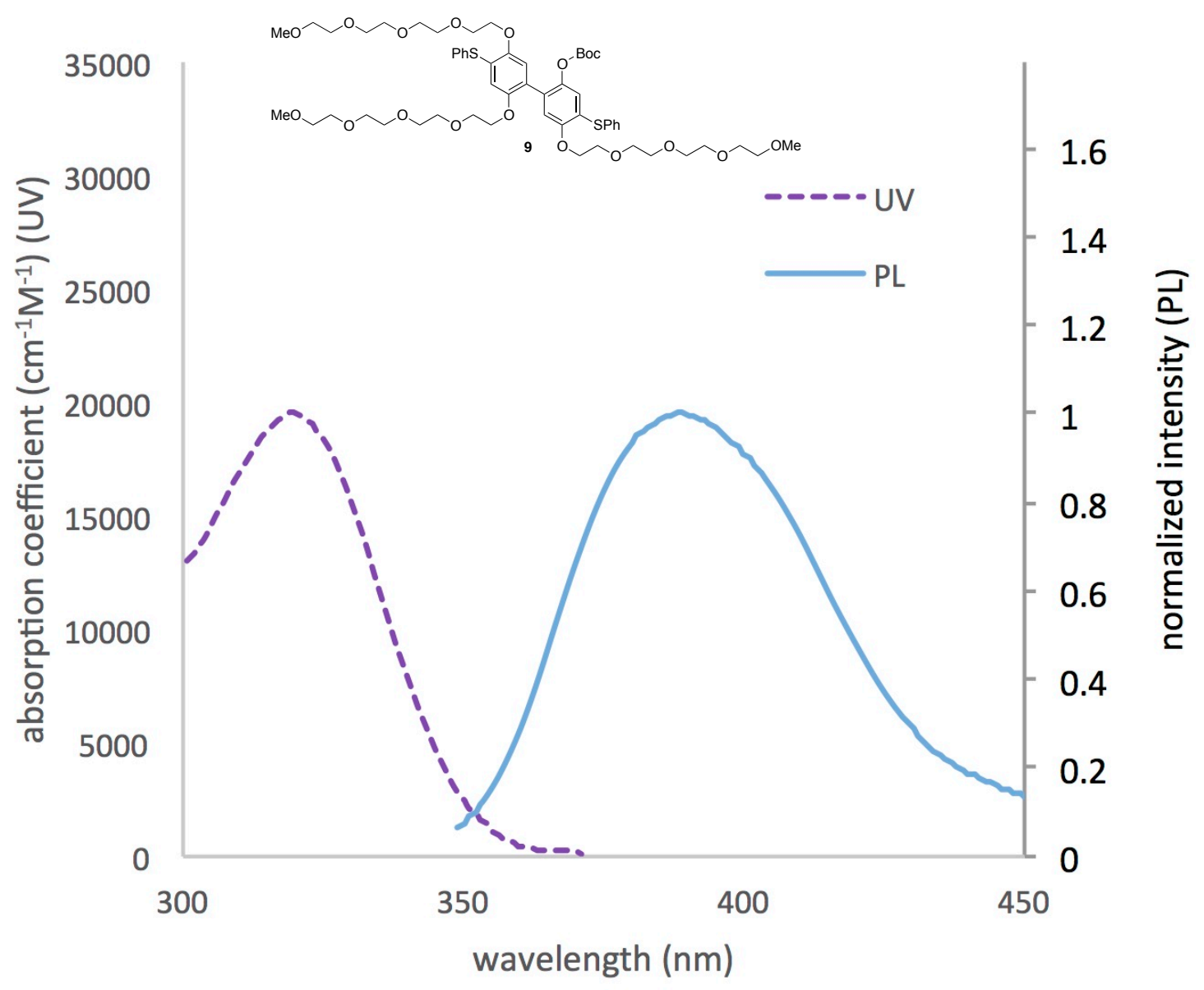

Figure S16. UV-PL spectra for compound 9 (in $\mathrm{MeOH}$ )

$\lambda_{\max }=319 \mathrm{~nm}, \varepsilon=2.0 \times 10^{4} \mathrm{M}^{-1} \mathrm{~cm}^{-1}, \lambda_{\mathrm{em}}=389 \mathrm{~nm}, \Phi=0.127$ 


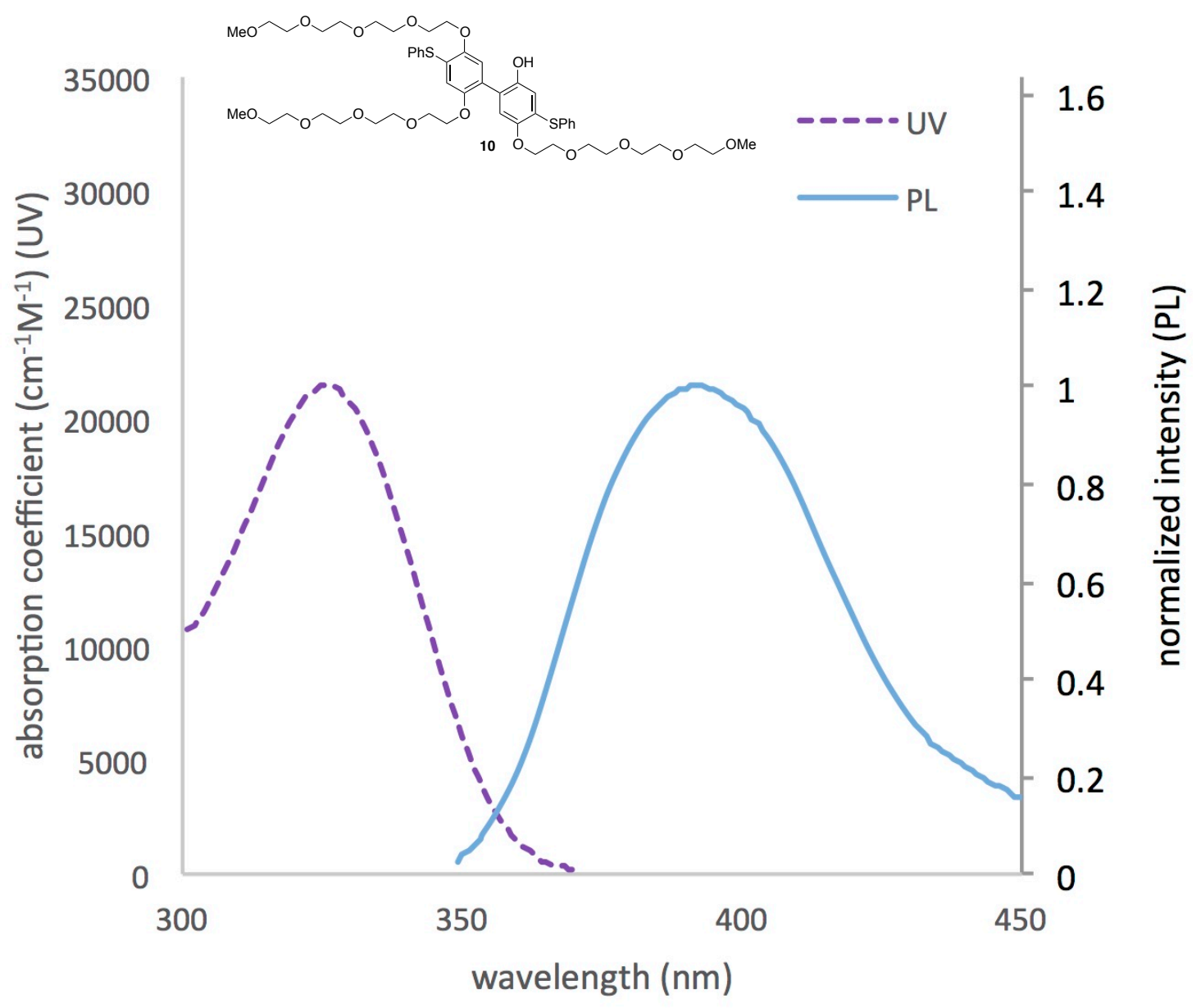

Figure S17. UV-PL spectra for compound 10 (in $\mathrm{MeOH}$ )

$\lambda_{\max }=325 \mathrm{~nm}, \varepsilon=2.1 \times 10^{4} \mathrm{M}^{-1} \mathrm{~cm}^{-1}, \lambda_{\mathrm{em}}=392 \mathrm{~nm}, \Phi=0.179$ 


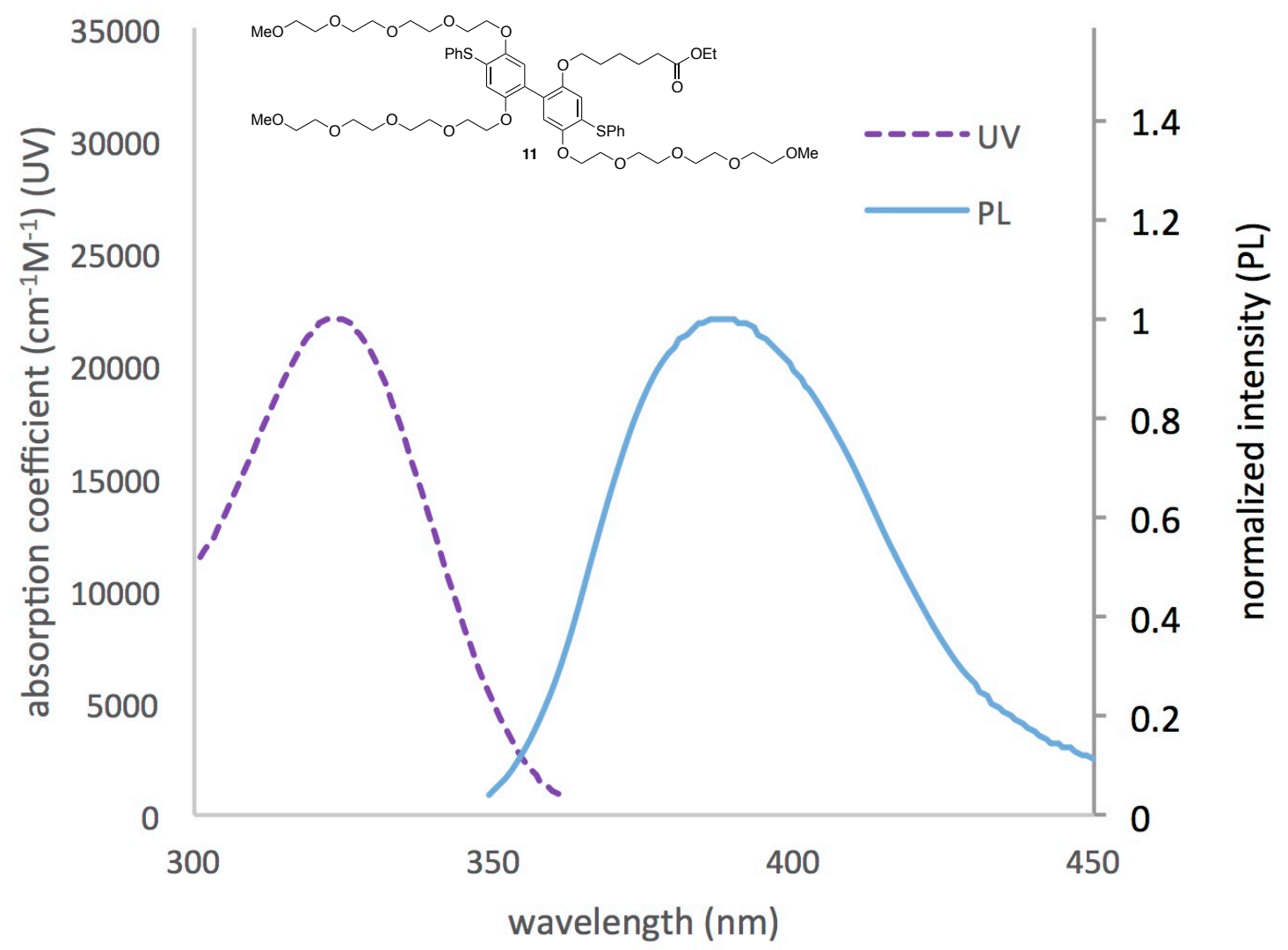

Figure S18. UV-PL spectra for compound 11 (in $\mathrm{MeOH}$ )

$\lambda_{\max }=322 \mathrm{~nm}, \varepsilon=2.2 \times 10^{4} \mathrm{M}^{-1} \mathrm{~cm}^{-1}, \lambda_{\mathrm{em}}=388 \mathrm{~nm}, \Phi=0.165$ 


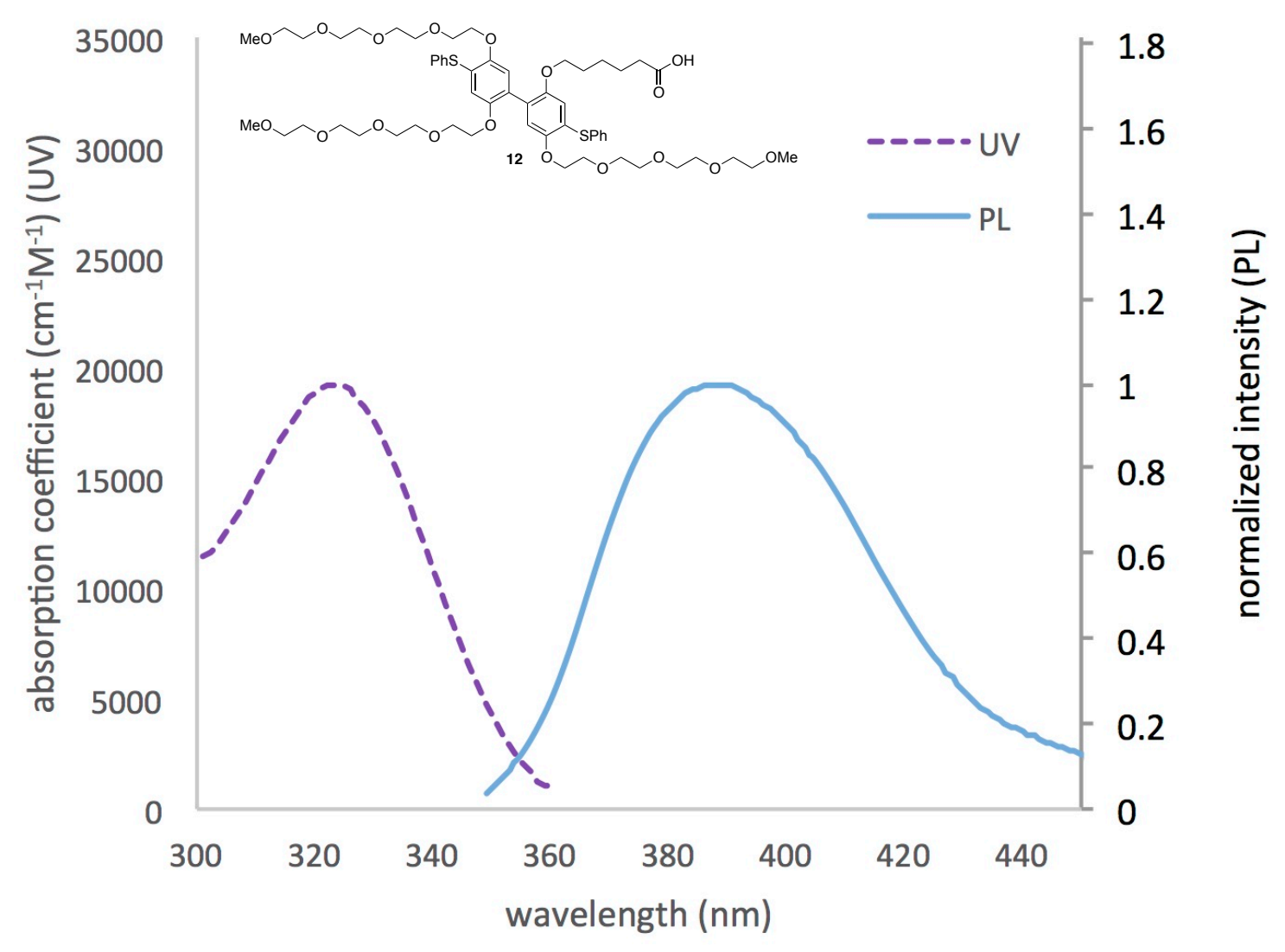

Figure S19. UV-PL spectra for compound 12 (in $\mathrm{MeOH}$ )

$\lambda_{\max }=322 \mathrm{~nm}, \varepsilon=2.0 \times 10^{4} \mathrm{M}^{-1} \mathrm{~cm}^{-1}, \lambda_{\mathrm{em}}=388 \mathrm{~nm}, \Phi=0.170$ 


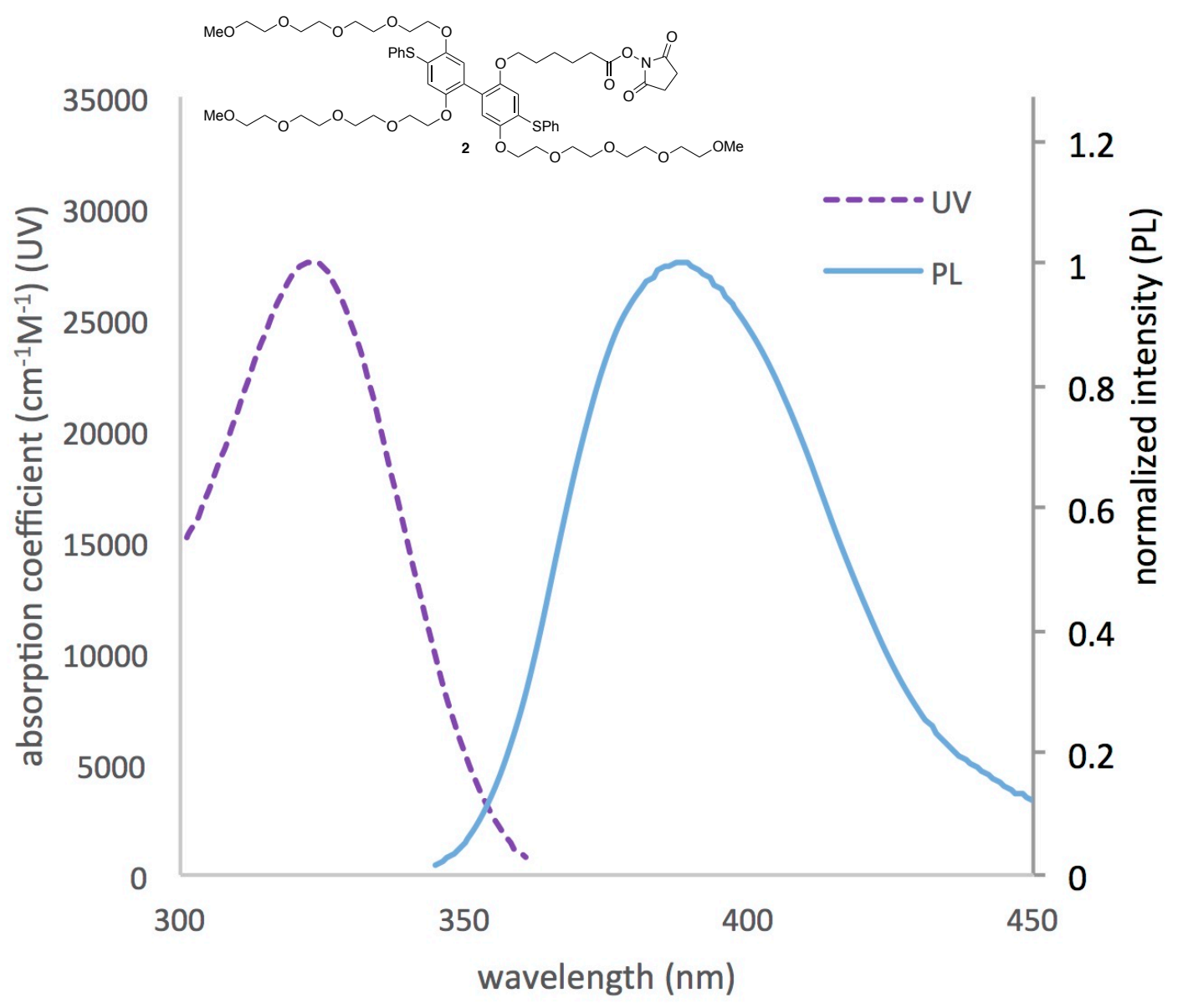

Figure S20. UV-PL spectra for compound 2 (in $\mathrm{MeOH}$ )

$\lambda_{\max }=322 \mathrm{~nm}, \varepsilon=2.2 \times 10^{4} \mathrm{M}^{-1} \mathrm{~cm}^{-1}, \lambda_{\mathrm{em}}=388 \mathrm{~nm}, \Phi=0.168$ 